\title{
The Influence of Structural Defects on the Martensitic Transformation
}

F.C. Lovey and V. Torra*

Centro Atómico Bariloche, San Carlos de Bariloche, Argentina

* Departament Física Aplicada, ETSECCPB, UPC, Barcelona, Spain

\begin{abstract}
Martensitic single interface transformations, induced by temperature or applied stresses in high resolution apparatus, show an intrinsic thermoelasticity or pseudoelasticity. This phenomenon arises from the interaction of the growing martensite with the preexisting dislocations in the parent phase. The intrinsic thermoelasticity has a great influence on the hysteretic behavior of the transformation, as it prevents the growth of a unique martensite plate. A further increase of the thermodynamically driving force favors the nucleation of new plates. Simulation of the hysteresis loops, when several plates are present, can be performed, in a more realistic way, by considering the various microscopic physical events taking place during the transformation: nucleation of the phases, interaction with the dislocations and stacking faults, number of martensite plates, hysteresis of each plate in single interface condition and interaction between the plates. The interaction of the martensitic transformation with small precipitates also produces an important influence on the hysteretic behavior: the hysteresis becomes larger but decreases gradually with transformation cycling. This behavior can be explained by considering the mechanisms of plastic accommodation of the precipitate in the martensite.
\end{abstract}

\section{INTRODUCTION}

The martensitic transformation between metastable phases is classically considered a first order phase transition occurring by nucleation and growth of the product phase. However, some characteristics of the transformation do no follow a pure first order behavior. A thermoelasticity is always observed, in such a way that the amount of transformed materials is a function of the undercooling with respect to the equilibrium temperature $T_{0}$. In addition, the nucleation and growth are associated to irreversible processes, giving rise to a hysteresis in the transformation cycles.

The thermoelasticity has been attributed to the elastic accommodation of the transformational shape change during the first stages of growth $[1,2]$ and, for subsequent stages, to the interaction between differently oriented variants of martensite $[3,4]$. In single interface transformation, where no elastic strain energy is stored, thermoelasticity has been assumed to be absent $[1,4,5]$. However, it has been shown, from very careful experiments, that the thermoelasticity can also be observed in single interface transformation $[6,7]$. This has been attributed to the interaction of the transformation with dislocations.

Other defect, like small particles of a second phase, has also a great influence on the properties of shape memory alloys. They increase the hysteresis of the transformation $[8,9]$ due to the plastic accommodation when embedded in the martensite [10] and favors the two way shape memory effects [11]. The basic interaction of the martensitic transformation with these structural defects will be 
reviewed and their effect on the hysteretic behavior will be described. It will be shown that the shape of the hysteresis loops and subloops in stress induced transformation can be simulated to a good agreement by considering the interaction with defects and the other elementary processes taking place during the transformation, as nucleation, interface friction, etc.

\section{THERMOELASTICITY IN SINGLE INTERFACE TRANSFORMATION}

Recent experiments of single interface transformations, in $\mathrm{Cu}-\mathrm{Zn}-\mathrm{Al}$ single crystals, have shown that the transformation does not occur at a constant temperature [7]. Moreover, it was found that the undercooling necessary to drive the transformation was dependent on the previous thermal treatment of the specimen. This is shown in Fig. 1 for air quenched (a) and water quenched (b) samples respectively. Some aspects of these results can be emphasized: i) The smallest measured hysteresis width was about $0.06 \mathrm{~K}$. ii) The transformation temperature is shifted to a lower value in the water quenched specimen. This is a permanent effect probably associated to the size of the ordered antiphase domains [12]. In addition the slopes of the curves shown in Fig. 1 do not change on cycling and they are independent

of the cooling rates. iv) The coefficient $\mu_{t h}=-\frac{d T(x)}{d x}$ gives a measure of the thermoelasticity: $\mu_{\mathrm{th}} \approx 0.004 \mathrm{~K}_{\mu \mathrm{m}^{-1}}$ and $\mu_{\mathrm{th}} \approx 0.05 \mathrm{~K} / \mathrm{m}^{-1}$ for air and water quenched specimens respectively.

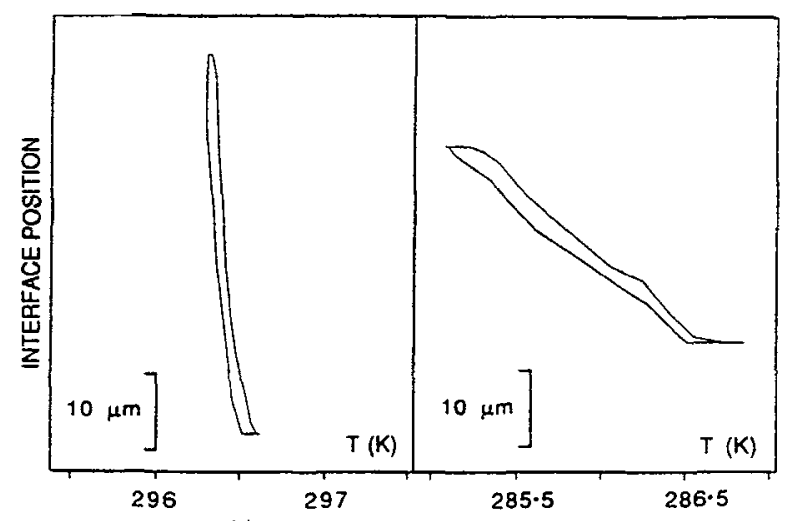

(a)

(b)

Figure 1: Hysteresis cycles of interface position $x$ against temperature $T$ : (a) after quenching in air, and (b) after water quenching.

It has been observed by transmission electron microscopy that the water quenched specimen had a higher dislocation density than the air quenched one. The Burgers vectors of the dislocations were all of the $\langle 100\rangle_{\beta}$ type. The origin of the thermoelasticity in Fig. 1 has been attributed to the interaction of the martensite with these dislocations [7], according with the following model: It can be observed in Fig. 2a and $2 \mathrm{~b}$ that the Burgers vector $[100]_{\beta}$ is changed into the $[100]_{\mathrm{R}}$ vector after the transformation, this latter vector posses translation symmetry in the $9 \mathrm{R}$ lattice. For encrgetic reasons a further dissociation into Shockley partials occurs [13]. For the $[010]_{\beta}$ and $[001]_{\beta}$ Burgers vectors the situation is different. Comparing figures $1 \mathrm{c}$ and $1 \mathrm{~d}$, it can be noted that such vectors are not well defined in the $9 \mathrm{R}$ lattice. In the martensite the vectors having translation symmetry are $B_{2}$ and $B_{3}$, which are nearly three times the original Burgers vectors. The inherited Burgers vectors in the $9 R$ structure can be taken as $b_{2}=B_{2} / 3$ and $b_{3}=B_{3} / 3$, respectively. None of them with translation symmetry [14].

On the average, for a given variant of martensite, the Burgers vectors of $2 / 3$ of the dislocations will transform into $b_{2}$ or $b_{3}$. Hence, stacking faults will be dragged by the moving interface on cooling. This 
phenomenon is schematically depicted in Fig. 3a. A transmission electron microscopy image of such fault is shown in Fig. 3b. Thus, as the interface advances, the new transformed slices are more and more faulted.

(a)

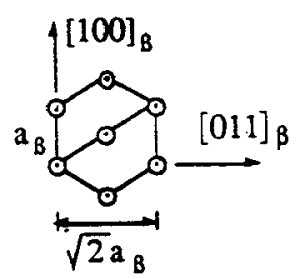

(c)

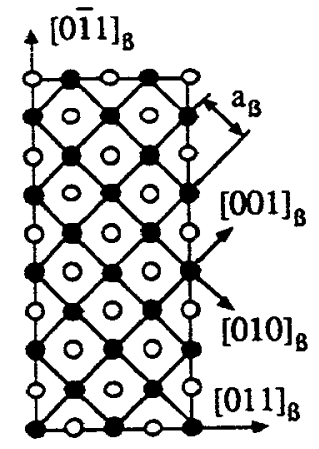

(b)

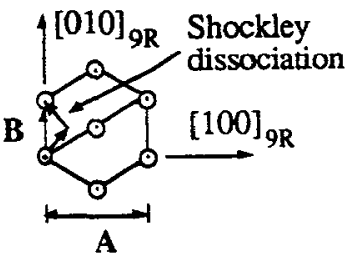

(d)

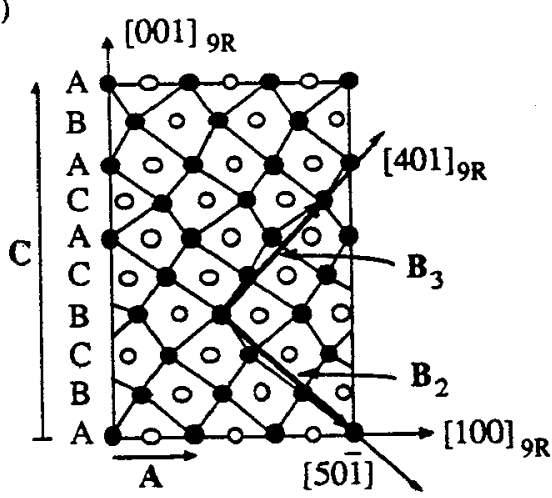

Figure 2: (a) the $(1 \overline{10})_{\beta}$ plane and (b) the corresponding martensite basal plane. In (c) the $(100)_{\beta}$ is shown and in (d) the corresponding (010) in martensite. Open and close circles represent atoms at different levels.

The free energy density of the martensite increases with $x$ by an amount given by [7] :

$$
\Delta g_{f} \approx \frac{\gamma \rho x}{2}
$$

where $\gamma$ is the stacking fault energy per area unit and $\rho$ is the density of the dislocations having Burgers vectors with no translation symmetry and line direction nearty parallel to the habit plane.

On the other hand the driving force to drive the transformation is provided by the undercooling as:

$$
\Delta g=\Delta T \Delta s
$$

where $\Delta s$ is the entropy difference per volume unit between the $\beta$ phase and the martensite. Combining equations (1) and (2) the thermoelastic coefficient reads:

$$
\mu_{\text {th }} \approx \frac{\gamma \rho}{2 \Delta \mathbf{s}}
$$

For water quenched specimens, the density of dislocations contributing to the intrinsic thermoelasticity is $\rho \approx 3.3 \times 10^{11} \mathrm{~m} \mathrm{~m}^{-3}$, and the corresponding amount of transformation is $\mathrm{x} \approx 26 \mu \mathrm{m}$. Taking 


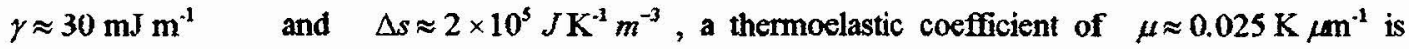
obtained. This result is in reasonable agreement with the experimental observation shown in Fig. $1 \mathrm{~b}$. The interaction of the martensite with the dislocations prevents the growth of a plate at constant temperature. When the undercooling increases other plates can nucleate and grow; this is the very reason for the appearance of a large number of plates in heterogeneous martensitic nucleation. The sizes of the plates are expected to be related to the dislocation density as it has been indeed observed [15]. In specimens having a few dislocations larger plates are formed and under special circumstances the shape memory effect can be broken, in the reverse transformation, by the appearance of the second variant of the parent phase. This second variant is related to the original one by the symmetry operations of the martensite [16].

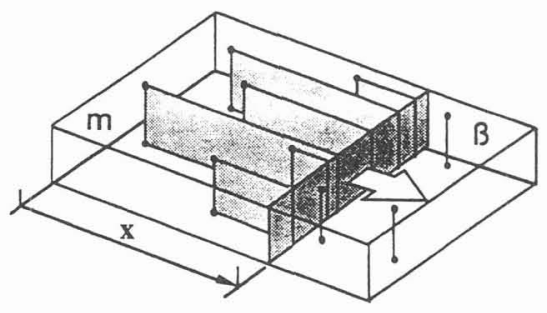

(a)

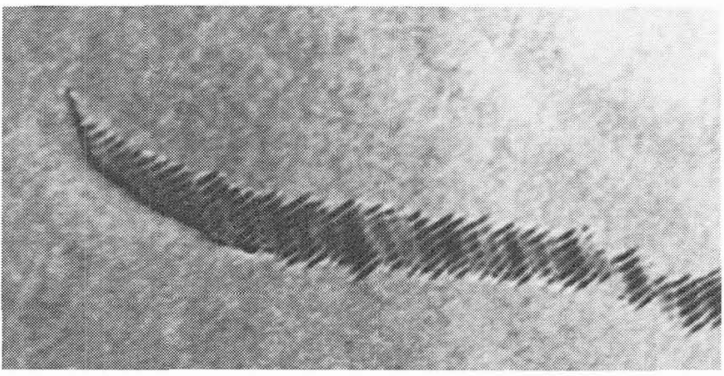

(b)

Figure 3: (a) Schematic representation of the stacking faults dragged by the moving interface. In (b) a transmission electron microscopy image of a fault originated from a dislocation is shown.

\section{STRESS INDUCED TRANSFORMATION AND HYSTERESIS LOOPS.}

The experiments were performed several days after the thermal treatment in order to permit ordering and vacancy annihilation. Thus the dependence of the characteristics of the hysteresis cycles on diffusional process is diminished. The stress-deformation curves have been obtained with a special developed apparatus with a resolution in the load and deformation of $1 \mathrm{mN}$ and $0.1 \mu \mathrm{m}$ respectively; the temperature of the specimen could be controlled within $0.01 \mathrm{~K}[17,18]$.

\subsection{Single interface transformation.}

A stress-deformation curve, corresponding to a single interface transformation in a air quenched specimen, is shown in Fig. 4a. In this experiment, when the critical stress is attained, only one martensite plate nucleates and grows, this occurs at the point 1 in Fig 4a. Since the applied load is kept nearly constant no yield point is observed in the nucleation process. After unloading to the point $\mathrm{x}_{1}$ and loading again, the plate grows in single interface condition along the curve indicated as 2; the applied stress has to be gradually increased in order to make the plate to grow. Such behavior was called "intrinsic pseudoelasticity" and arises from the interaction of the transformation with dislocations. The

coefficient of the intrinsic pseudoelasticity can be defined as $\mu_{\mathrm{px}}=2 \frac{\mathrm{d} \tau}{\mathrm{dx}}$, where $\mathrm{x}$ is the plate width, being related to $\Delta l$ by the magnitude of the macroscopic shear deformation and the tensile axis 
orientation ( $x=6.8 \Delta l$ for the experiment of Fig $4 a)$; the factor 2 appears because two interfaces are moving.

The value of $\Delta \tau_{h}$ gives the hysteresis associated to the interface movement. On the other hand $\Delta \tau_{n}$ corresponds to the nucleation process, i.e., the excess of applied stress to initiate the transformation with respect to the stress necessary to transform in single interface condition.

\subsection{The hysteretic behavior when several plates of the same variant are present.}

In water quenched specimen the density of dislocations is higher [7] and so it is the intrinsic pseudoelasticity. This prevents the growth of only one plate, leading to the successive appearance of other plates as the stress is gradually increased. A typical behavior of the stress-deformation curve is shown in Fig. 4b. The slope $\mathrm{d} \tau / \mathrm{dx}(\mathrm{x}=6 \mathrm{dl})$ does not represent the intrinsic pseudoelasticity because it depends on the number of plates [17]. The hysteresis width of the largest cycle in Fig. $4 \mathrm{~b}$ is similar to the largest one in Fig. 4a, suggesting that the hysteresis in this case is mainty attached to the nucleation processes. The loops of partial cycles are inside those corresponding to the larger cycles, as observed usually in martensitic transformations [4]. The complex behavior of the stress deformation curves in Fig. 4 is the result of a combination of several cycles similar to that shown in paragraph 3.1.

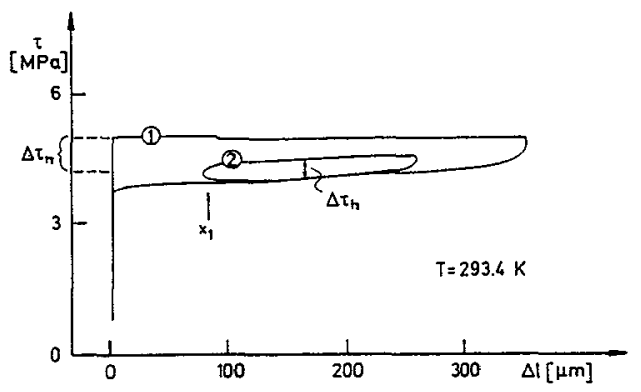

(a)

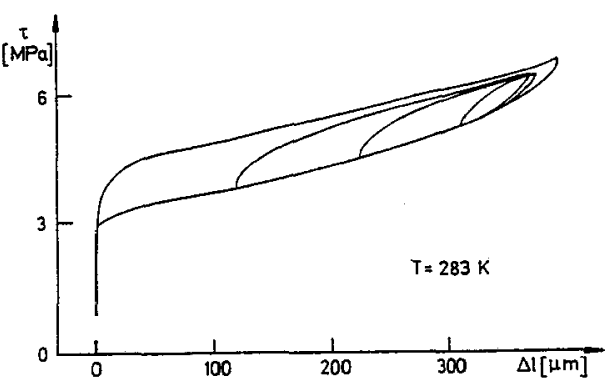

(b)

Figure 4: Resolved shear stress, $\tau$, as a function of elongation, $\Delta$, for: (a) single interface transformation, and (b) when several plates are present.

\section{MODELING OF THE STRESS INDUCED HYSTERESIS CYCLES}

In order to simulate the hysteresis loops shown in Fig. 4, the following procedure was adopted. Each martensite plate was assumed to behave as schematically shown in Fig $5 a$ or $5 b$. The value of $x$, in Fig 5 , indicates the plate width, which is related to the deformation $\Delta$ by the Schmid factor and the macroscopic shear deformation. The basic parameters to be defined for a given $i^{\text {th }}$ plate are: i) The critical stress $\tau_{\mathrm{c}}^{(i)}$ for the appearance of the plate, including nucleation. ii) The average hysteresis width, $\Delta \tau_{\mathrm{h}}^{(\mathrm{i})}$, of the single interface transformation . iii) The slope, $\mu_{\mathrm{ps}}$, of the cycle in the single interface condition. iv) The stress, $\tau_{0}^{(1)}$, giving the thermodynamical equilibrium between the phases. $k$ ) In cases where the plates have joined each other on loading, nucleation of the $\beta$ phase would be needed in the 
retransformation. This will require a decreasing of the stress by an amount $\Delta t_{\beta}^{(i)}$. vi) The lowest stress, $\tau_{d}^{\text {(i) }}$, necessary for the complete disappearance of the plate.

On loading, the martensite plate will suddenly transform up to a thickness $\Delta x^{(i)}$, as soon as the applied stress, $\tau$, attains the value $\tau_{\mathrm{c}}^{(\mathrm{i})}$, where $\Delta \mathrm{x}^{(\mathrm{i})}=\frac{\Delta \tau_{\mathrm{n}}^{(\mathrm{i})}}{\mu_{\mathrm{ps}}}$ and $\Delta \tau_{\mathrm{n}}^{(\mathrm{i})}=\tau_{\mathrm{c}}^{(\mathrm{i})}-\left(\tau_{0}^{(\mathrm{i})}+\frac{1}{2} \Delta \tau_{\mathrm{b}}^{(\mathrm{i})}\right)$

The conditions for the plate growth, at different values of $\tau$, can be written as follows:

$$
\begin{array}{ll}
\mathrm{x}^{(\mathrm{i})}=0, & \text { if } \tau<\tau_{\mathrm{c}}^{(\mathrm{i})} \\
\mathrm{x}^{(\mathrm{i})}=\Delta \mathrm{x}^{(\mathrm{i})}, & \text { if } \tau=\tau_{c}^{(\mathrm{i})} \\
\mathrm{x}^{(\mathrm{i})}=\Delta \mathrm{x}^{(\mathrm{i})}+\frac{1}{\mu_{\mathrm{ps}}}\left(\tau-\tau_{\mathrm{c}}^{(\mathrm{i})}\right), & \text { if } \tau>\tau_{\mathrm{c}}^{(\mathrm{i})}
\end{array}
$$

The amount of transformation after the appearance of $\mathrm{N}$ martensite plates is given by:

$$
x_{t}=\sum_{i=1}^{N} x^{(i)}
$$

with the condition $x^{(i)} \cap x^{(j)} \equiv \varnothing$, i.e. no overlapping of the plates is allowed.

A similar reasoning can be applied during the unloading process, as indicated in Fig. 5a and $\mathrm{b}$. Fig. 5c shows the shape of the curve $\tau-x$ due to the contribution of two plates.

Using the basic algorithm depicted in Fig. 5, the shape of the loops and subloops, when several plates are present, were computer simulated. This is shown in Fig. 6 for the following values of the various parameters:

$\mathrm{N}=40, \quad \Delta \tau_{\mathrm{n}}^{(\mathrm{i})}=1 \mathrm{MPa}, \quad \tau_{0}^{(\mathrm{D})}=\left(\tau_{0}^{(1)}+0.05 \mathrm{i}\right) \mathrm{MPa}, \quad \Delta \tau_{\mathrm{h}}^{(\mathrm{i})}=\left(0.3+\delta^{(\mathrm{i})}\right), \quad \Delta \tau_{\beta}^{(i)}=1 \mathrm{MPa} \quad$ and $\Delta \tau_{\mathrm{d}}^{(\mathrm{i})}=1 \mathrm{MPa}$.

From the results, several aspects can be emphasized:

- The slope of the stress deformation curve is related to the inverse of the number of moving or active interfaces. The true intrinsic pseudoelasticity can be obtained when only one plate is moving (stage 1 , in Fig. 6). The slope diminishes gradually as more and more plates nucleate and growth (stage 2). If no more interval for the transformation of a given plate is avalaible, the number of moving interfaces decreases, giving rise to an increase of the slope (stage 3). This explains the typical shape of the curve during the transformation

- On unloading, the inverse process occurs. Once the external stress is reduced by an amount $\Delta \tau_{h}$, the interfaces which are free move backwards (stage 4). After nucleation of some $\beta$ slices, the number of moving interfaces increases and the slope is again reduced (stage 5). At the end of the re-transformation several plates have arrived to their thinnest width and the slope increases again (stage 6).

- When the transforming specimen is partially unloading and loading again the interfaces, which were moving backwards, start moving in the opposite direction as soon as the applied stress has been increased by an amount $\Delta \tau_{\mathrm{h}}$. Since most of the plates are still present, some of them in the thinnest stage (with $\tau>\tau_{\mathrm{d}}$ ), no nucleation is needed, as a consequence the stresses to have the same amount of transformed material remain lower those of the external cycles. 

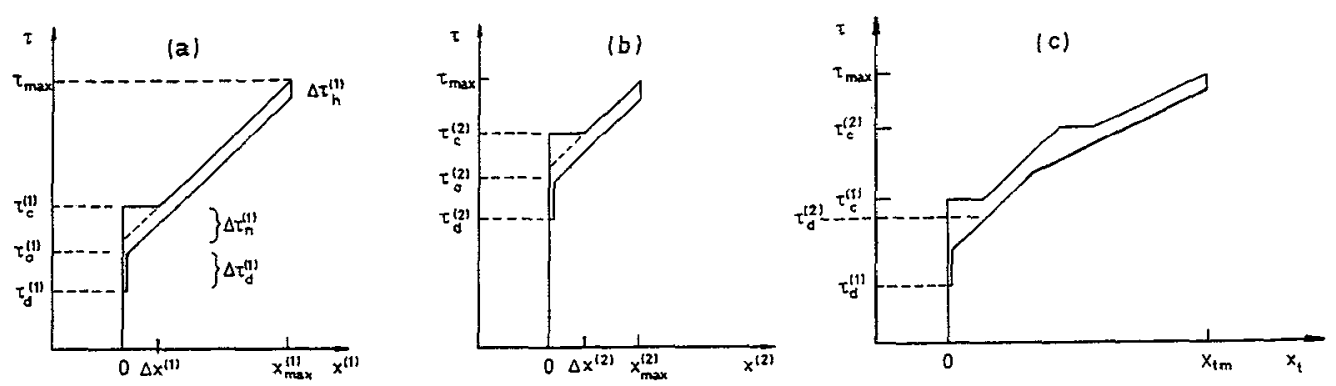

Figure 5: Schematic representation of the $\tau$-x curve. (a) First plate, (b) second plate and (c) the behavior of the $\tau-x_{t}$ when both plates are present, $x_{t}=x^{(1)}+x^{(2)}$.

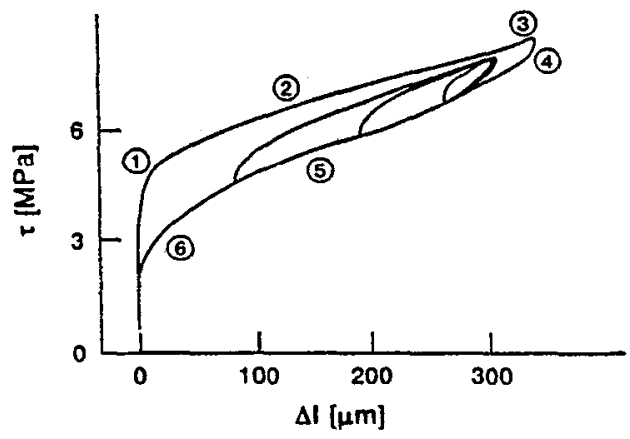

Figure 6: Computer simulated stress-deformation cycles based on the intrinsic parameters experimentally measured

\section{EFFECT OF $\gamma$ PRECIPITATES ON SINGLE VARIANT TRANSFORMATION.}

Small $\gamma$ type precipitates, with an average size of $7 \mathrm{~nm}$ and a density of $\delta \approx 8 \times 10^{21} \mathrm{prec} . / \mathrm{m}^{3}$, can be introduced in $\mathrm{Cu}-\mathrm{Zn}$-Al alloys by the following thermal treatment [19]: Solution treatment at $1120 \mathrm{~K}$ for $10 \mathrm{~min}$, then cooling to $800 \mathrm{~K}$ followed by quenching in cold water. The temperature induced single interface transformation shows a wider hysteresis for the samples containing precipitates [10] as shown in Fig. 7. The hysteresis diminishes with the number of cycles mainly due to the increase of the transformation temperature. The encircled numbers in Fig. 7, indicate the times that area was cycled.

The decreasing of the hysteresis as a function of the transformation cycles was found to be similar either for temperature or stress induced transformations.

The main interaction of the precipitates with the martensite was suggested to arise from the shape change of the $\beta$-matrix cavities where the precipitates are embedded [10]. Results of the calculations using the W-L-R theory [20] is shown in Fig. 9. The value of $x$ in Fig.9 is about $5 \%$ the precipitate length, i.e., far from any elastic accommodation process. 


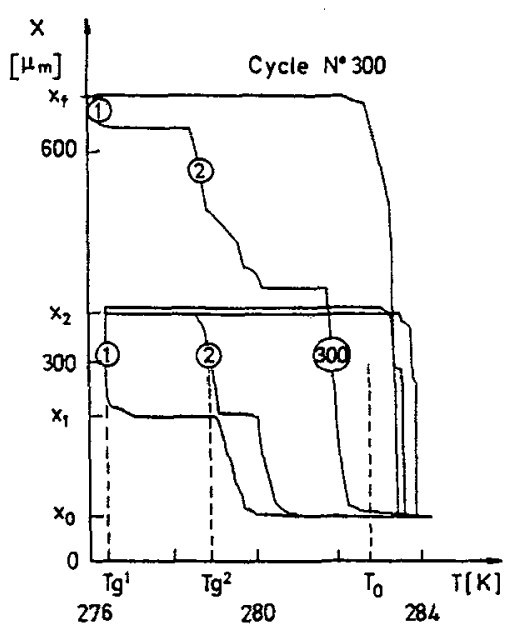

Figure 7: Single interface transformation in a sample containing precipitates. Variation of the hysteresis width on transformation cycling. The encircled numbers indicate the number of times that area, $x$, of the specimen was cycled.

The decrease of the hysteresis in the first cycles has been attributed to the creation of dislocations necessary for the plastic accommodation, whilst the asymptotic value of the hysteresis (about $1.5 \mathrm{~K}$ ) has been related to the plastic accommodation itself.

An estimate of the driving force necessary to produce the plastic accommodation has been done in terms of the indentation theory [21]. The precipitates have been assumed to be undeformable, thus the excess of material in the regions indicated as $\mathrm{C}$ in Fig. 9 are plastically removed towards the areas indicated as $\mathrm{E}$.

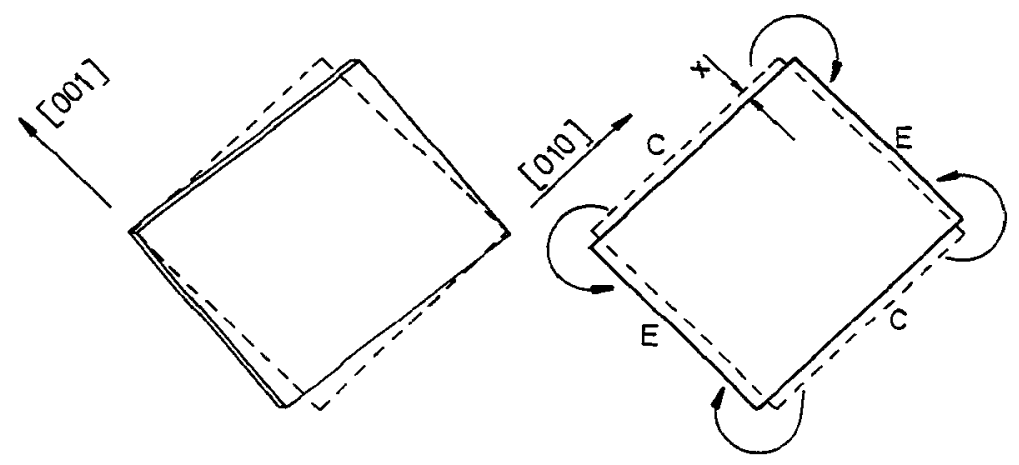

(a)

(b)

Figure 8. (a) Change of the cubic matrix cavity (dashed line) with the transformation. (b) Insertion of the cubic precipitate (dashed lines) into the deformed hole (thick lines). 
The energy loss in piercing the precipitate into a depth $\mathrm{x}$ is given by:

$$
W \approx 2 \mathrm{~A} \tau \mathrm{c} \mathrm{x}
$$

where $A$ is the area of the precipitate faces, $\tau$ is the critical resolved shear stress (CRSS) for plastic deformation and $c$ is a geometrical factor which varies from about 3 to about 5.2, depending on the geometry of the problem, the amount of work hardening, and the yielding criteria [21]. Concerning the CRSS $\tau$, the deformation can occur partially in martensite and partially in the $\beta$ phase as the precipitate is being absorbed by the martensite. Hence, the average value between $\tau_{\beta} \approx 200 \mathrm{MPa}$ [22] and $\tau_{m} \approx 80 \mathrm{MPa}[23]$ will be taken for further calculations.

Using the upper value of $c$ in (6),

$$
W \approx 2.5 \times 10^{-17} \mathrm{~J} / \text { prec. }
$$

In thermally induced transformation the driving force per precipitate is provided by the undercooling as:

$$
\Delta g=\frac{\Delta \mathrm{T} \Delta \mathrm{s}}{\delta}
$$

where $\Delta \mathrm{s} \approx 2 \times 10^{5} \mathrm{~J} / \mathrm{K} \mathrm{m}^{3}$ is the entropy difference per volume unit between $\beta$ and martensite.

Using $\Delta T \approx 0.75 \mathrm{~K}$, corresponding to the half of the asymptotic value of the hysteresis, equation (8) gives:

$$
\Delta g \approx 1.8 \times 10^{-17} \mathrm{~J} / \mathrm{prec} .
$$

The driving force given by (9) is in good agreement with the plastic work obtained in (7). A similar process would occur during the retransformation, accounting for the other half of the hysteresis.

\section{CONCLUSIONS}

1) The intrinsic thermoelasticity or pseudoelasticity in single interface transformations arises from the loss of translation symmetry of the dislocation Burgers vectors when absorbed by the martensite.

2) This interaction of the martensite with the dislocations prevents the growth of a unique plate, leading to the appearance of a large number of plates.

3) The characteristics of the hysteresis cycles can be computer simulated in terms of the intrinsic parameters: thermoelasticity and interface friction in single interface transformation, nucleation processes (parent to martensite and vice-versa) and number of martensite plates.

4) The presence of precipitates enhances the hysteresis which decreases on cycling. Evolutive behavior. implies dislocations creation. The plastic accommodation, due to the shape change associated to the transformation, gives the residual hysteresis.

\section{ACKNOWLEDGMENTS:}

The work was partially supported by the DGICYT of Spain (sabatic program), CICYT MAT92-1025C02-01, the Commission of European Communities under contract ALAMED CI1*-CT91-0943 and the CONICET of Argentina.

\section{REFERENCES:}

[1] Oison G.A. and Cohen M., Scripta metall., 9 (1975) 1247-1254.

[2] Ling H.C. and Owen, W.S., Acta metall., 29 (1981) 1721-1736.

[3] Salzbrener R. J. and Cohen M., Acta metall., 27 (1979) 739-748.

[4] Delaey L. and Arnoudt E., Proceedings of the International Conference on Martensitic

Transformation (Nara: Japan Institut of Metals), (1986) 926-933.

[5] Wollants P. Roos J.R. and Delaey L., Prog. Mater. Sci., 37 (1993) 227-288. 
[6] Amengual A., Garcias F., Marco F., Segui C. and Torra V., Acta metall., 36 (1988) 2329-2334.

[7] Lovey F.C., Amengual A., Torra V. and Ahlers M., Phil. Mag. A, 61 (1990) 159-165.

[8] Auguet C., Cesari E., Rapacioli R. and Mañosa Ll., Scripta metall., 23 (1989) 579-583.

[9] Lovey F.C., Cesari E., Auguet C., Mañosa L1. and Rapacioli R., Mater. Sci. Forum, 56-58 (1990) 493-498.

[10] Lovey F.C., Torra V., Isalgue A., Roqueta D. and Sade M., Acta metall. mater., 42 (1994) 453-460.

[11] Cesari E., C. Picornell, J. Pons and M. Sade, J. Physique IV, 1(C4) (1991) 451-456.

[12] Rapacioli R. and Ahlers M., Acta metall., 27 (1979) 777.

[13] Rodriguez P.L., Cuniberti A., Romero R. and Lovey F.C., Scr. metall. mater., 27 (1992) 1133-1138.

[14] Lovey F.C., Sade M., Torra V. and Amengual A., Proceedings of the International Conference on Martensitic Transformations (Monterey, Eds.: Wayman C.M. and Perkins J.), (1992) 365-370.

[15] Pons J., Cesari E. and Lovcy F.C., Acta metall. mater., 38 (1990) 2733-2740.

[16] Lovey F.C., Amengual A. and Torra V.,Phil. Mag. A, 64 (1991) 787-796.

[17] Lovey F.C., Isalgue A. and Torra V., Acta metall. mater., 40 (1992) 3389-3394.

[18] Amengual A., Isalgue A., Marco F., Tachoire H., Torra V. and Torra V. R., J. Therm. Anal., 38 (1992) 583-592.

[19] Lovey F.C., Rapacioli R. and Chandrasekaran M., Physica status solidi (a), 68 (1981) K 105-108

[20] Wechsler M.S., Lieberman D.S. and Read T.A., Trans. Am. Inst. Min. Engrs., 197 (1953) 1503.

[21] Hill R., The Mathematical Theory of Plasticity, Clarendon Press, Oxford (1956), p. 254.

[22] Romero R., Lovey F.C. and Ahlers M., Phil. Mag. A, 58 (1988) 881.

[23] Cuniberti A., Romero R. and Ahlers M., Scripta metall. mater., 26 (1992) 495. 